Article

\title{
Prime and irreducible filters in strong quasi-ordered residuated systems
}

\section{Daniel A. Romano}

International Mathematical Virtual Institute 6, Kordunaška Street, 78000 Banja Luka, Bosnia and Herzegovina; bato49@hotmail.com

Academic Editor: Elena Guardo

Received: 2 January 2021; Accepted: 13 April 2021; Published: 19 April 2021.

Abstract: In this article, we continue our research on quasi-ordered residuated systems introduced in 2018 by S. Bonzio and I. Chajda and various types of filters in them. Some fundamental properties of strong quasi-ordered residuated systems are given in this article. In addition, the concepts of prime and irreducible filters in such systems are introduced and analyzed.

Keywords: Quasi-ordered residuated system; Strong quasi-ordered residuated system; Prime and irreducible filters.

MSC: 08A02; 06A11; 06F05.

\section{Introduction}

$\mathbf{T}$ he concept of residuated relational systems ordered under a quasi-order relation, or quasi-ordered residuated systems (briefly, QRS), was introduced in 2018 by S. Bonzio and I. Chajda [1]. Previously, this idea was discussed in [2,3]. The author introduced and developed the concepts of filters [4] in this algebraic structure as well as several types of filters such as implicative [5], associated [6], comparative [7] and weak implicative filters [8]. In [7], it is shown that every comparative filter of a quasi-ordered residuated system $\mathfrak{A}$ is an implicative filter of $\mathfrak{A}$ and the reverse it need not be valid. The concept of a strong quasi-ordered residuated system was introduced and discussed in [9]. In such systems, comparative and implicative filters coincide. The specificity of strong QRSs is that they allow us to determine the least upper bound for each their two elements.

In this paper, as a continuation of the previous research, we analyse the fundamental properties of the least upper bound in such systems (Theorem 4 and Proposition 2). In addition, the concepts of prime (Definition 9) and irreducible (Definition 10) filters in strong quasi-ordered residuated systems will be introduced and some their important properties will be recognized (Theorem 5, Theorem 6 and Theorem 7). It is shown (Theorem 9) that any prime filter in a strong quasi-ordered residuated system is an irreducible filter.

\section{Preliminaries}

\subsection{Concept of quasi-ordered residuated systems}

In article [1], S. Bonzio and I. Chajda introduced and analyzed the concept of residual relational systems.

Definition 1 ([1], Definition 2.1). A residuated relational system is a structure $\mathfrak{A}=\langle A, \cdot, \rightarrow, 1, R\rangle$, where $\langle A, \cdot, \rightarrow$ $, 1\rangle$ is an algebra of type $\langle 2,2,0\rangle$ and $R$ is a binary relation on $A$ and satisfying the following properties:

(1) $(A, \cdot, 1)$ is a commutative monoid;

(2) $(\forall x \in A)((x, 1) \in R)$;

(3) $(\forall x, y, z \in A)((x \cdot y, z) \in R \Longleftrightarrow(x, y \rightarrow z) \in R)$.

We will refer to the operation - as multiplication, to $\rightarrow$ as its residuum and to condition (3) as residuation.

The basic properties for residuated relational systems are subsumed in the following:

Theorem 1 ([1], Proposition 2.1). Let $\mathfrak{A}=\langle A, \cdot, \rightarrow, 1, R\rangle$ be a residuated relational system. Then 
(4) $(\forall x, y \in A)(x \rightarrow y=1 \Longrightarrow(x, y) \in R)$;

(5) $(\forall x \in A)((x, 1 \rightarrow 1) \in R)$;

(6) $(\forall x \in A)((1, x \rightarrow 1) \in R)$;

(7) $(\forall x, y, z \in A)(x \rightarrow y=1 \Longrightarrow(z \cdot x, y) \in R)$;

(8) $(\forall x, y \in A)((x, y \rightarrow 1) \in R)$.

Recall that a quasi-order relation ${ }^{\prime} \preccurlyeq{ }^{\prime}$ on a set $A$ is a binary relation which is reflexive and transitive.

Definition 2 ([1]). A quasi-ordered residuated system is a residuated relational system $\mathfrak{A}=\langle A, \cdot, \rightarrow, 1$, $\rangle$, where $\preccurlyeq$ is a quasi-order relation in the monoid $(A, \cdot)$.

Example 1. Let $A=\{1, a, b, c, d\}$ and operations '.' and ' $\rightarrow$ ' defined on $A$ as follows:

\begin{tabular}{c|ccccc}
$\cdot$ & 1 & $\mathrm{a}$ & $\mathrm{b}$ & $\mathrm{c}$ & $\mathrm{d}$ \\
\hline $\mathrm{l}$ & 1 & $\mathrm{a}$ & $\mathrm{b}$ & $\mathrm{c}$ & $\mathrm{d}$ \\
$\mathrm{a}$ & $\mathrm{a}$ & $\mathrm{a}$ & $\mathrm{d}$ & $\mathrm{c}$ & $\mathrm{d}$ \\
$\mathrm{b}$ & $\mathrm{b}$ & $\mathrm{d}$ & $\mathrm{b}$ & $\mathrm{d}$ & $\mathrm{d}$ \\
$\mathrm{c}$ & $\mathrm{c}$ & $\mathrm{c}$ & $\mathrm{d}$ & $\mathrm{c}$ & $\mathrm{d}$ \\
$\mathrm{d}$ & $\mathrm{d}$ & $\mathrm{d}$ & $\mathrm{d}$ & $\mathrm{d}$ & $\mathrm{d}$
\end{tabular}

\begin{tabular}{c|ccccc}
$\rightarrow$ & 1 & $\mathrm{a}$ & $\mathrm{b}$ & $\mathrm{c}$ & $\mathrm{d}$ \\
\hline 1 & 1 & $\mathrm{a}$ & $\mathrm{b}$ & $\mathrm{c}$ & $\mathrm{d}$ \\
$\mathrm{a}$ & 1 & 1 & $\mathrm{~b}$ & $\mathrm{c}$ & $\mathrm{d}$ \\
$\mathrm{b}$ & 1 & $\mathrm{a}$ & 1 & $\mathrm{c}$ & $\mathrm{c}$ \\
$\mathrm{c}$ & 1 & 1 & $\mathrm{~b}$ & 1 & $\mathrm{~b}$ \\
$\mathrm{~d}$ & 1 & 1 & 1 & 1 & 1
\end{tabular}

Then $\mathfrak{A}=\langle A, \cdot, \rightarrow, 1\rangle$ is a quasi-ordered residuated systems where the relation ' $\preccurlyeq$ ' is defined as follows

$$
\preccurlyeq:=\{(1,1),(a, 1),(b, 1),(c, 1),(d, 1),(b, b),(a, a),(c, c),(d, d),(c, a),(d, a),(d, b),(d, c)\} .
$$

Example 2. For a commutative monoid $A$, let $\mathfrak{P}(A)$ denote the powerset of $A$ ordered by set inclusion and '. ' the usual multiplication of subsets of $A$. Then $\langle\mathfrak{P}(A), \cdot, \rightarrow, A, \subseteq\rangle$ is a quasi-ordered residuated system in which the residuum are given by

$$
(\forall X, Y \in \mathfrak{P}(A))(Y \rightarrow X:=\{z \in A: Y z \subseteq X\}) .
$$

Example 3. Let $\mathbb{R}$ be a field of real numbers. Define a binary operations '.' and ' $\rightarrow$ ' on $A=[0,1] \subset \mathbb{R}$ by

$$
(\forall x, y \in[0,1])(x \cdot y:=\max \{0, x+y-1\}) \text { and } x \rightarrow y:=\min \{1,1-x+y\}) .
$$

Then, $A$ is a commutative monoid with the identity 1 and $\langle A, \cdot, \rightarrow,<, 1\rangle$ is a quasi-ordered residuated system.

Example 4. Let $A=\langle-\infty, 1] \subset \mathbb{R}$ (the real numbers field). If we define '.' and ' $\rightarrow$ ' as follows, $(\forall u, v \in$ $A)(u \cdot v:=\min \{u, v\})$ and $u \rightarrow v:=1$ if $u \leqslant v$ and $u \rightarrow v:=v$ if $v<u$ for all $u, v \in A$, then $\mathfrak{A}:=\langle A, \cdot, \rightarrow, 1,<\rangle$ is a quasi-ordered residuated system.

Example 5. Any commutative residuated lattice $\langle A, \cdot, \rightarrow, 0,1, \sqcap, \sqcup, R\rangle$ where $R$ is a lattice quasi-order is a quasi-ordered residuated system.

Example 6. ([10], Example 2.2) Any commutative residuated po-monoid (briefly, a CRPM) $(A, \cdot, \rightarrow, 1, \leqslant)$ is a

\begin{tabular}{|c|c|c|c|c|c|c|c|c|c|c|c|c|c|c|}
\hline & 1 & a & $\mathrm{b}$ & C & $\mathrm{d}$ & $\mathrm{e}$ & & $\rightarrow$ & 1 & a & $b$ & c & $\mathrm{d}$ & e \\
\hline 1 & 1 & $a$ & $\mathrm{~b}$ & $\mathrm{c}$ & $\mathrm{d}$ & $\mathrm{e}$ & & 1 & 1 & $a$ & $\mathrm{~b}$ & c & $\mathrm{d}$ & $\mathrm{e}$ \\
\hline a & a & $\mathrm{b}$ & $\mathrm{a}$ & c & d & $\mathrm{e}$ & & $\mathrm{a}$ & 1 & 1 & c & 1 & 1 & 1 \\
\hline $\mathrm{b}$ & $\mathrm{b}$ & $b$ & $\mathrm{a}$ & c & d & $\mathrm{e}$ & and & b & 1 & $c$ & 1 & 1 & 1 & 1 \\
\hline c & c & $b$ & $c$ & c & d & $\mathrm{e}$ & & c & 1 & c & c & 1 & 1 & 1 \\
\hline $\mathrm{d}$ & d & $\mathrm{d}$ & d & d & $\mathrm{e}$ & $\mathrm{e}$ & & d & 1 & d & d & $\mathrm{d}$ & 1 & $\mathrm{e}$ \\
\hline e & $\mathrm{e}$ & $\mathrm{e}$ & $\mathrm{e}$ & $\mathrm{e}$ & $\mathrm{e}$ & d & & e & 1 & $\mathrm{e}$ & $\mathrm{e}$ & e & $\mathrm{d}$ & 1 \\
\hline
\end{tabular}
quasi-ordered system.

Example 7. Let $A=\{1, a, b, c, d, e\}$ and operations '.' and ' $\rightarrow$ ' defined on $A$ as follows:

Then $\mathfrak{A}=\langle A, \cdot \rightarrow, 1\rangle$ is a quasi-ordered residuated systems, where the relation ' $\preccurlyeq$ ' is defined as follows $\preccurlyeq:=\{(1,1),(a, 1),(b, 1),(c, 1),(d, 1),(e, 1),(a, a),(a, c),(a, d),(a, e),(b, b),(b, c),(b, d),(b, e),(c, c),(c, d),(c, e)$, $(d, d),(e, e)\}$. 
It should be noted that the elements $a$ and $b$ are not comparable.

Remark 1. Quasi-ordered residauted system, generally speaking, differs from the commutative residuated lattice $\langle A, \cdot, \rightarrow, 0,1, \sqcap, \sqcup, R\rangle$ where $R$ is a lattice quasi- order. First, our observed system does not have to be limited from below. Second, the observed system does not have to be a lattice. However, the difference between a quasi-ordered relational system and a CRPM (Example 6) is only in order relations since a quasi-order relation does not have to be antisymmetric. Some more about this last-mentioned algebraic structure it can be found in [11].

The following proposition shows the basic properties of quasi-ordered residuated systems.

Proposition 1 ([1], Proposition 3.1). Let A be a quasi-ordered residuated system. Then

(9) $(\forall x, y, z \in A)(x \preccurlyeq y \Longrightarrow(x \cdot z \preccurlyeq y \cdot z \wedge z \cdot x \preccurlyeq z \cdot y))$;

(10) $(\forall x, y, z \in A)(x \preccurlyeq y \Longrightarrow(y \rightarrow z \preccurlyeq x \rightarrow z \wedge z \rightarrow x \preccurlyeq z \rightarrow y))$;

(11) $(\forall x, y \in A)(x \cdot y \preccurlyeq x \wedge x \cdot y \preccurlyeq y)$.

\subsection{Concept of filters}

In this subsection we give some notions that will be used in this article. In a well-known book [12], H. Rasiowa used the term 'implicative filter' if the subset $F$ in an implicative algebra $\mathfrak{A}$ satisfies conditions (F0) and (F3) (see below in the text). This determination is somewhat different from our determination given by the Definition 3. In addition, Rasiowa designs some other 'special' filters in the so-called implicative algebras.

Definition 3 ([4], Definition 3.1). For a non-empty subset $F$ of a quasi-ordered residuated system $\mathfrak{A}$ we say that it is a filter of $\mathfrak{A}$ if it satisfies the following conditions

(F2) $(\forall u, v \in A)((u \in F \wedge u \preccurlyeq v) \Longrightarrow v \in F)$, and

(F3) $(\forall u, v \in A)((u \in F \wedge u \rightarrow v \in F) \Longrightarrow v \in F)$.

It is shown ([4], Proposition 3.4 and Proposition 3.2) that if a non-empty subset $F$ of a quasi-ordered system $\mathfrak{A}$ satisfies the condition (F2), then it also satisfies the conditions

(F0): $1 \in F$ and

(F1): $(\forall u, v \in A)((u \cdot v \in F \Longrightarrow(u \in F \wedge v \in F))$.

If $\mathfrak{F}(A)$ is the family of all filters in a QRS $\mathfrak{A}$, then $\mathfrak{F}(A)$ is a complete lattice ([4], Theorem 3.1).

Example 8. Let $A=\{1,2,3,4\}$ and operations '.' and ' $\rightarrow$ ' defined on $A$ as follows:

\begin{tabular}{c|cccc}
$\cdot$ & 1 & 2 & 3 & 4 \\
\hline 1 & 1 & 2 & 3 & 4 \\
2 & 2 & 2 & 2 & 4 \\
3 & 3 & 2 & 2 & 4 \\
4 & 4 & 4 & 4 & 4
\end{tabular}$\quad$ and $\quad$\begin{tabular}{ll|llll}
$\rightarrow$ & 1 & 2 & 3 & 4 \\
\hline 1 & 1 & 2 & 3 & 4 \\
2 & 1 & 1 & 1 & 1 \\
3 & 1 & 2 & 1 & 4 \\
4 & 1 & 2 & 3 & 1
\end{tabular}

Then $\mathfrak{A}=\langle A, \cdot, \rightarrow, 1\rangle$ is a quasi-ordered residuated systems where the relation ' $\preccurlyeq$ ' is defined as follows

$$
\preccurlyeq=\{(1,1),(2,2),(3,3),(4,4),(4,1),(3,1),(2,1),(2,3),(2,4)\} .
$$

Then the subsets $\{1\},\{1,3\}$ and $\{1,3,4\}$ are filters in $\mathfrak{A}$.

Remark 2. In implicative algebras, the term 'implicative filter' is used instead of the term 'filter' we use (see, for example [12,13]). It is obvious that our filter concept is also a filter in the sense of [12-14]. The term 'special implicative filter' is also used in the aforementioned sources if the implicative filter in the sense of [12] satisfies some additional condition.

There is considerable diversity in the literature in the use of terms that cover additional conditions that are met by filters (see, for example [15-17]). The terms we opted for our previous papers [5,7] and for use in 
this paper are taken from [16,17]. We have developed the concepts of implicative, associated, comparative and weak implicative filters in quasi-ordered residuated systems:

Definition 4 ([5]). For a non-empty subset $F$ of a quasi-ordered residuated system $\mathfrak{A}$ we say that the implicative filter of $\mathfrak{A}$ if (F2) and the following condition

(IF) $(\forall u, v, z \in A)((u \rightarrow(v \rightarrow z) \in F \wedge u \rightarrow v \in F) \Longrightarrow u \rightarrow z \in F)$

are valid.

Example 9. In Example 7, filter $\{1,3\}$ is an implicative filter in $\mathfrak{A}$.

Definition 5. ([6]) Let $\mathfrak{A}$ be a QRS and $x$ be a fixed element of $A$. A non-empty subset $F$ of $A$ is called an associated filter of $\mathfrak{A}$ with respect to $x$ (briefly, $x$-associated filter of $\mathfrak{A}$ ) if it satisfies the condition (F2) and

(FA) $(\forall y, z \in A)((x \rightarrow(y \rightarrow z) \in F \wedge x \rightarrow y \in F) \Longrightarrow z \in F)$

By an associated filter of $\mathfrak{A}$, we mean an $x$-associated filter of $\mathfrak{A}$ for all $x$ in $A$.

Example 10. Let $A=\{1,2,3,4\}$ and operations '.' and ' $\rightarrow$ ' defined on $A$ as follows:

\begin{tabular}{c|cccc}
$\cdot$ & 1 & 2 & 3 & 4 \\
\hline 1 & 1 & 2 & 3 & 4 \\
2 & 2 & 2 & 3 & 4 \\
3 & 3 & 3 & 3 & 4 \\
4 & 4 & 4 & 4 & 4
\end{tabular}$\quad$ and $\quad$\begin{tabular}{ll|llll}
$\rightarrow$ & 1 & 2 & 3 & 4 \\
\hline 1 & 1 & 2 & 3 & 4 \\
2 & 1 & 1 & 2 & 3 \\
3 & 1 & 1 & 1 & 4 \\
4 & 1 & 1 & 1 & 1
\end{tabular}

Then $\mathfrak{A}=\langle A, \cdot, \rightarrow, 1\rangle$ is a quasi-ordered residuated systems where the relation ' $\preccurlyeq$ ' is defined as follows

$$
\preccurlyeq=\{(1,1),(2,2),(3,3),(4,4),(4,1),(3,1),(2,1),(3,2),(4,2),(4,3)\} .
$$

Then $\{1\}$ is a 1-associated filter of $\mathfrak{A}$. The subset $\{1,2,3\}$ are associated in $\mathfrak{A}$ with respect to 1,2 and 3 , but it is not a 4 -associated filter of $\mathfrak{A}$ because $4 \rightarrow(4 \rightarrow 4)=4 \rightarrow 1=1 \in\{1,2,3\}$ and $4 \rightarrow 4=1 \in\{1,2,3\}$ but $\neg(4 \in\{1,2,3\})$.

Definition 6 ([7]). For a non-empty subset $F$ of a quasi-ordered residuated system $\mathfrak{A}$ we say that a comparative filter of $\mathfrak{A}$ if (F2) and the following condition

(FC) $(\forall u, v, z \in A)((u \rightarrow((v \rightarrow z) \rightarrow v) \in F \wedge u \in F) \Longrightarrow v \in F)$

are valid.

Example 11. Let $\mathfrak{A}$ be a quasi-ordered residuated system as in Example 1 . Then the set $F:=\{1, a, b\}$ is a comparative filter in $\mathfrak{A}$.

Definition 7. ([8]) For a non-empty subset $F$ of a quasi-ordered residuated system $\mathfrak{A}$ we say that the weak implicative filter in $\mathfrak{A}$ if (F2) and the following condition

(WIF) $(\forall u, v, z \in A)((u \rightarrow(v \rightarrow z) \in F \wedge u \rightarrow v \in F) \Longrightarrow u \rightarrow(u \rightarrow z) \in F)$

are valid.

Example 12. Let $A=\{1, a, b, c, d\}$ and operations '.' and ' $\rightarrow$ ' defined on $A$ as follows:

\begin{tabular}{|c|c|c|c|c|c|c|c|c|c|c|c|c|}
\hline$\cdot$ & 1 & a & $b$ & C & d & \multirow{4}{*}{ and } & $\rightarrow$ & 1 & a & b & c & $\mathrm{d}$ \\
\hline 1 & 1 & $a$ & $b$ & C & d & & 1 & 1 & $a$ & $b$ & C & $\mathrm{d}$ \\
\hline $\mathrm{a}$ & $\mathrm{a}$ & a & $\mathrm{a}$ & $\mathrm{a}$ & b & & $\mathrm{a}$ & 1 & 1 & $b$ & c & $b$ \\
\hline $\mathrm{b}$ & $\mathrm{b}$ & a & $b$ & $\mathrm{~b}$ & $\mathrm{~d}$ & & $\mathrm{~b}$ & 1 & $\mathrm{a}$ & 1 & $b$ & $\mathrm{a}$ \\
\hline C & C & a & $b$ & C & $\mathrm{d}$ & & c & 1 & $\mathrm{a}$ & 1 & 1 & a \\
\hline d & $\mathrm{d}$ & $b$ & d & d & $\lambda$ & & $\mathrm{d}$ & 1 & 1 & 1 & $b$ & 1 \\
\hline
\end{tabular}


Then $\mathfrak{A}=\langle A, \cdot, \rightarrow, 1\rangle$ is a quasi-ordered residuated systems where the relation ' $\preccurlyeq$ ' is defined as follows

$$
\preccurlyeq=\{(1,1),(a, 1),(b, 1),(c, 1),(d, 1),(a, a),(b, b),(c, c),(d, d),(d, a),(d, b),(c, b)\} .
$$

Then the subsets $\{1, a\}$ is a weak implicative filter in $\mathfrak{A}$.

Notions and notations that are used but not previously determined in this paper can be found in [1,4-7].

\section{The main results}

\subsection{Some fundamental properties of strong QRS}

In this subsection we analyze the concept of strong quasi-ordered residuated systems. This concept was introduced and analyzed in [9].

Considering the fact that the quasi-order relation ' $\preccurlyeq$ ', which appears in the determination of this algebraic system, does not have to be antisymmetric, the following definition gets a clearer meaning. It is generally known that a quasi-order relation $\preccurlyeq$ on a set $A$ generates an equivalence relation $\equiv_{\preccurlyeq}: \preccurlyeq \curvearrowleft \preccurlyeq^{-1}$ on $A$. Due to properties (9) and (10), this equivalence is compatible with the operations in $\mathfrak{A}$. Thus, the relation $\equiv_{\preccurlyeq}$ is a congruence on $\mathfrak{A}$.

Definition 8. ([9]) For a quasi-ordered residuated system $\mathfrak{A}$ it is said to be a strong quasi-ordered residuated system if the following holds

(14) $(\forall u, v \in A)\left((u \rightarrow v) \rightarrow v \equiv_{\preccurlyeq}(v \rightarrow u) \rightarrow u\right)$.

Remark 3. Note that if

$$
(\forall u, v \in A)((u \rightarrow v) \rightarrow v=(v \rightarrow u) \rightarrow u)
$$

is valid, then (14) is also valid. The reverse does not have to be true.

The following is an example of a non strong QRS:

\begin{tabular}{|c|c|c|c|c|c|c|c|c|}
\hline & 1 & $\mathrm{a}$ & $\mathrm{b}$ & \multirow{3}{*}{ and } & $\rightarrow$ & 1 & a & $b$ \\
\hline 1 & 1 & $\mathrm{a}$ & $\mathrm{b}$ & & 1 & 1 & $a$ & $\mathrm{~b}$ \\
\hline $\mathrm{a}$ & a & $\mathrm{a}$ & $\mathrm{a}$ & & $\mathrm{a}$ & 1 & 1 & 1 \\
\hline$b$ & $\mathrm{~b}$ & $\mathrm{a}$ & $\mathrm{b}$ & & $b$ & 1 & a & 1 \\
\hline
\end{tabular}

Example 13. Let $A=\{1, a, b\}$ and the operations '.' and ' $\rightarrow$ ' be defined on $A$ as follows:

Then $\mathfrak{A}=\langle A, \cdot, \rightarrow, 1\rangle$ is a quasi-ordered residuated systems where the relation ' $\preccurlyeq$ ' is defined as follows

$$
\preccurlyeq:=\{(1,1),(a, a),(b, b),(a, 1),(b, 1),(a, b)\} .
$$

It can be easily checked that $\mathfrak{A}$ is a quasi-ordered residuated system. Since

$$
(a \rightarrow b) \rightarrow b=1 \rightarrow b=b \text { and }(b \rightarrow a) \rightarrow a=a \rightarrow a=1,
$$

we have $(a \rightarrow b) \rightarrow b \preccurlyeq(b \rightarrow a) \rightarrow a$ but $\neg((b \rightarrow a) \rightarrow a \preccurlyeq(a \rightarrow b) \rightarrow b)$. Thus, $\mathfrak{A}$ is not a strong

\begin{tabular}{|c|c|c|c|c|c|c|c|c|c|c|}
\hline 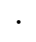 & 1 & a & b & C & & $\rightarrow$ & 1 & a & b & C \\
\hline 1 & 1 & $\mathrm{a}$ & $b$ & C & & 1 & 1 & a & $b$ & C \\
\hline $\mathrm{a}$ & $\mathrm{a}$ & a & a & a & and & $\mathrm{a}$ & 1 & 1 & 1 & 1 \\
\hline b & $\mathrm{b}$ & a & b & a & & $\mathrm{b}$ & 1 & C & 1 & C \\
\hline C & C & $a$ & $\mathrm{a}$ & C & & C & 1 & $\mathrm{~b}$ & b & 1 \\
\hline
\end{tabular}
quasi-ordered residuated system.

Now, we give an example of strong quasi-ordered residuated system.

Example 14. Let $A=\{1, a, b, c\}$ and operations '.' and ' $\rightarrow$ ' defined on $A$ as follows: 
Then $\mathfrak{A}=\langle A, \cdot \rightarrow, 1\rangle$ is a quasi-ordered residuated systems where the relation ' $\preccurlyeq{ }^{\prime}$ is defined as follows

$$
\preccurlyeq:=\{(1,1),(a, 1),(b, 1),(c, 1),(a, a),(b, b),(c, c),(a, b),(a, c)\} .
$$

Direct verification it can prove that $\mathfrak{A}$ is a strong quasi-ordered residuated system.

In this paper, we shall investigate the structure of strong quasi-ordered residuated systems. In [9], it is shown that comparative and implicative filters in such algebraic systems coincide. Some of the propositions made in this article have already been shown in [9] such as Theorem 2, Theorem 3 and Theorem 4 . We are re-enclose them in this subsection in order to achieve greater consistency of the material presented in it.

Theorem 2 ([9]). Let $\mathfrak{A}$ be a strong quasi-ordered residuated system. Then the following holds

(15) $(\forall u, v \in A)\left(u \preccurlyeq v \Longrightarrow v \equiv_{\preccurlyeq}(v \rightarrow u) \rightarrow u\right)$.

Proof. Assume that $\mathfrak{A}$ is a strong quasi-ordered system. For given $u, v \in A$, let be $u \preccurlyeq v$. First, as $v \preccurlyeq(u \rightarrow$ $v) \rightarrow v$ is valid according to (11), from here we immediately get the following

$$
v \preccurlyeq(u \rightarrow v) \rightarrow v \preccurlyeq(v \rightarrow u) \rightarrow u .
$$

by (14). Second, from $u \preccurlyeq v$ it follows $1 \preccurlyeq u \rightarrow v$ by (3). From here, applying (10), we obtain $(u \rightarrow v) \rightarrow v \preccurlyeq$ $1 \rightarrow v$. Thus

$$
(v \rightarrow u) \rightarrow u \equiv \preccurlyeq(u \rightarrow v) \rightarrow v) \preccurlyeq 1 \rightarrow v
$$

with respect (14). Applying the claim (d) of Proposition 2.3 in [1], from here we get $(v \rightarrow u) \rightarrow u \preccurlyeq v$. This proves that $u \preccurlyeq v \Longrightarrow v \equiv_{\preccurlyeq}(v \rightarrow u) \rightarrow u$ is valid.

Corollary 1. Let $\mathfrak{A}$ be a strong quasi-ordered residuated system. Then the following holds

(16) $(\forall x, y \in A)\left(y \rightarrow x \equiv_{\preccurlyeq}((y \rightarrow x) \rightarrow x) \rightarrow x\right)$ and

(17) $(\forall x, y \in A)\left((y \rightarrow x) \rightarrow x \equiv_{\preccurlyeq}(((y \rightarrow x) \rightarrow x) \rightarrow x) \rightarrow x\right)$.

Proof. For arbitrary elements $x, y \in A$ holds $x \preccurlyeq y \rightarrow x$ by (11). Thus $y \rightarrow x \equiv \preccurlyeq((y \rightarrow x) \rightarrow x) \rightarrow x$ by (15).

Proof of the claim (17) can be obtained in the same way as the proof for (16) since $x \preccurlyeq(y \rightarrow x) \rightarrow x$ is valid.

The following theorem shows that in a strong quasi-ordered residuated system we can construct the least upper bound for each pair of elements.

Theorem 3 ([9]). Let $\mathfrak{A}$ be a strong quasi-ordered residuated system. For any $u, v \in A$, the element

$$
u \sqcup v:=(v \rightarrow u) \rightarrow u \equiv \preccurlyeq(u \rightarrow v) \rightarrow v
$$

is the least upper bound of $u$ and $v$.

Proof. It is clear that the following hold

$$
\begin{aligned}
& u \preccurlyeq(v \rightarrow u) \rightarrow u=u \sqcup v \text { and } \\
& v \preccurlyeq(u \rightarrow v) \rightarrow v \equiv \preccurlyeq(v \rightarrow u) \rightarrow u=u \sqcup v
\end{aligned}
$$

by (11) and (14). This shows that $u \sqcup v$ is the upper bound for $u$ and $v$. If $z$ is any common upper bound of $u$ and $v$, then by Theorem 2 ,

$$
z \equiv_{\preccurlyeq}(z \rightarrow u) \rightarrow u \text { and } z \equiv_{\preccurlyeq}(z \rightarrow v) \rightarrow v .
$$

On the other hand, from $v \preccurlyeq(z \rightarrow v) \rightarrow v$ it follows

$$
((z \rightarrow v) \rightarrow v) \rightarrow u \preccurlyeq v \rightarrow u
$$

and

$$
(v \rightarrow u) \rightarrow u \preccurlyeq(((z \rightarrow v) \rightarrow v) \rightarrow u) \rightarrow u \equiv_{\preccurlyeq}(z \rightarrow u) \rightarrow u \equiv_{\preccurlyeq} z
$$

according to (10) and (15). Therefore, $u \sqcup v$ is the least upper bound of $u$ and $v$ in the system $\mathfrak{A}$. 
Theorem 4 ([9]). Let $\mathfrak{A}$ be a strong quasi-ordered residuated system. Then $(\mathfrak{A}, \sqcup)$ is a distributive upper semi-lattice in the following sense

$$
(\forall x, y, z \in A)\left((x \sqcup y) \sqcup z \equiv_{\preccurlyeq}(x \sqcup z) \sqcup(y \sqcup z)\right) .
$$

Proof. Let $x, y, z$ be arbitrary elements of a strong quasi-ordered residuated system. From $x \preccurlyeq x \sqcup z$ and $y \preccurlyeq y \sqcup z$ it follows $x \sqcup y \preccurlyeq(x \sqcup z) \sqcup(y \sqcup z)$. At the other hand, we have $z \preccurlyeq(x \sqcup z) \sqcup(y \sqcup z)$. Thus

$$
(x \sqcup y) \sqcup z \preccurlyeq(x \sqcup z) \sqcup(y \sqcup z) .
$$

Conversely, from $x \preccurlyeq x \sqcup y \preccurlyeq(x \sqcup y) \sqcup z$ and $z \preccurlyeq(x \sqcup y) \sqcup z$ it follows $x \sqcup z \preccurlyeq(x \sqcup y) \sqcup z$. Analogous to the previous one, we can get $y \sqcup z \preccurlyeq(x \sqcup y) \sqcup z$. Hence

$$
(x \sqcup z) \sqcup(y \sqcup z) \preccurlyeq(x \sqcup y) \sqcup z .
$$

This proves the theorem.

Remark 4. The term 'distributive upper semi-lattice' used here differs from the concept of 'distributive join semi-lattice' which appears for example in the book [18] (pp. 99).

Proposition 2. Let $\mathfrak{A}$ be a strong quasi-ordered residuated system. Then:

(a) $(\forall u, v \in A)(u \sqcup 1=1 \sqcup u=1$ and $u \sqcup v=v \sqcup u)$,

(b) $(\forall x, y, z \in A)((z \cdot x) \sqcup(z \cdot y) \preccurlyeq x \sqcup y)$,

(c) $(\forall x, y, z \in A)((x \sqcup y) \rightarrow z \preccurlyeq(x \rightarrow z) \sqcup(y \rightarrow z))$,

(d) $(\forall x, y, z \in A)((z \rightarrow x) \sqcup(z \rightarrow y) \preccurlyeq z \rightarrow(x \sqcup y))$,

(e) $(\forall, y \in A)(x \sqcup y \preccurlyeq(y \rightarrow x) \sqcup(x \rightarrow y))$,

(f) $(\forall x, y \in A)((x \sqcup y) \sqcup x \equiv \preccurlyeq x \sqcup y)$.

Proof. The proof of these equations (a) immediately follows from the determination of the concept of least upper bound with respect to (2).

Let $x, y, z \in A$ be arbitrary elements in a strong QRS $\mathfrak{A}$. It is obvious that $(z \cdot x) \sqcup(z \cdot y) \preccurlyeq x \sqcup y$ it follows from the valid inequalities $z \cdot x \preccurlyeq x \preccurlyeq x \sqcup y$ and $z \cdot y \preccurlyeq y \preccurlyeq x \sqcup y$.

From $x \preccurlyeq x \sqcup y$ and $y \preccurlyeq x \sqcup y$, it follows $(x \sqcup y) \rightarrow z \preccurlyeq x \rightarrow z$ and $(x \sqcup y) \rightarrow z \preccurlyeq y \rightarrow z$ by (10). Thus $(x \sqcup y) \rightarrow z \preccurlyeq(x \rightarrow z) \sqcup(y \rightarrow z)$.

It is obvious that $z \rightarrow x \preccurlyeq z \rightarrow(x \sqcup y)$ and $z \rightarrow y \preccurlyeq z \rightarrow(x \sqcup y)$ it follows from the valid inequalities $x \preccurlyeq x \sqcup y$ and $y \preccurlyeq x \sqcup y$ by (10). Thus $(z \rightarrow x) \sqcup(z \rightarrow y) \preccurlyeq z \rightarrow(x \sqcup u)$ according to the definition of the least upper bound.

The inequality $x \sqcup y \preccurlyeq(y \rightarrow x) \sqcup(x \rightarrow y)$ is obtained directly from the valid inequalities $x \preccurlyeq y \rightarrow x$ and $y \preccurlyeq x \rightarrow y$.

The claim (f) is the claim (17) written differently by using the notation ' $\sqcup$ '.

Example 15. Let $A$ be as in Example 7. By direct checking it can be determined that $(A, \cdot, \rightarrow)$ is a strong quasi-ordered system. For example, $a \sqcup b=(a \rightarrow b) \rightarrow b=(b \rightarrow a) \rightarrow a=c$ is valid. The semi-lattice $(A, \sqcup)$ is not a lattice since there is no lower bound for elements $a$ and $b$.

\subsection{Prime filters in QRS}

The following definition gives the concept of prime filters in QRSs.

Definition 9. Let $F$ be a filter of a strong quasi-ordered residuated system $\mathfrak{A}$. Then $F$ is said to be a prime filter in $\mathfrak{A}$ if the following holds

(PF) $(\forall u, v \in A)(u \sqcup v \in F \Longrightarrow(u \in F \vee v \in F))$.

Example 16. Let $A=\{1, a, b, c\}$ and operations '.' and ' $\rightarrow$ ' defined on $A$ as follows: 


\begin{tabular}{|c|c|c|c|c|c|c|c|c|c|c|}
\hline • & 1 & $\mathrm{a}$ & b & C & & $\rightarrow$ & 1 & $a$ & b & C \\
\hline 1 & 1 & $\mathrm{a}$ & $\mathrm{b}$ & C & & 1 & 1 & $\mathrm{a}$ & $b$ & C \\
\hline$a$ & $a$ & $\mathrm{a}$ & $\mathrm{a}$ & $a$ & and & $\mathrm{a}$ & 1 & 1 & 1 & 1 \\
\hline$b$ & b & $\mathrm{a}$ & $a$ & $a$ & & $b$ & 1 & C & 1 & 1 \\
\hline C & C & b & $\mathrm{a}$ & a & & C & 1 & $b$ & C & 1 \\
\hline
\end{tabular}

Then $\mathfrak{A}=\langle A, \cdot, \rightarrow, 1\rangle$ is a quasi-ordered residuated systems where the relation ' $\preccurlyeq$ ' is defined as follows

$$
\preccurlyeq:=\{(1,1),(a, 1),(b, 1),(c, 1),(a, a),(b, b),(c, c),(a, b),(a, c),(b, c)\} .
$$

Direct verification it can prove that $\mathfrak{A}$ is a strong quasi-ordered residuated system. The only proper filter in this system is the subset $F:=\{1\}$. It is easily concluded by directly checking that $F$ is a prime filter.

Example 17. Let $\mathfrak{A}$ be as in the Example 14. Then the subsets $\{1\},\{1, b\}$ and $\{1, c\}$ are filters in $\mathfrak{A}$. It can be checked that $F_{1}:=\{1, b\}$ and $F_{2}:=\{1, c\}$ are prime filters in $\mathfrak{A}$ while the filter $\{1\}$ is not prime because we have $b \sqcup c=1 \in\{1\}$ but $b \notin\{1\}$ and $c \notin\{1\}$.

We first show one important feature of prime filters in strong QRSs.

Proposition 3. Let $F$ be a prime filter of a strong quasi-ordered residuated system $\mathfrak{A}$. Then

$$
(\forall x, y \in A)(x \sqcup y \in F \Longrightarrow(x \rightarrow y \in F \vee y \rightarrow x \in F)) .
$$

Proof. The proof of this proposition follows directly from the claim (e) of Proposition 2, (F2) and (PF).

A sufficient criterion for a filter to be a prime filter in a strong QRS gives the following theorem.

Theorem 5. If a filter $F$ in a strong quasi-ordered residuated system $\mathfrak{A}$ satisfies the condition

(E) $(\forall x, y \in A)(x \rightarrow y \in F \vee y \rightarrow x \in F)$,

then $F$ is a prime filter in $\mathfrak{A}$.

Proof. Let $\mathfrak{A}$ be a strong quasi-ordered residuated system and assume that a filter $F$ in $\mathfrak{A}$ satisfies the condition (E). Let $x \sqcup y \in F$ be holds for elements $x, y \in A$, i.e. let $(x \rightarrow y) \rightarrow y \equiv \preccurlyeq(y \rightarrow x) \rightarrow x \in F$ be holds. Then from $x \rightarrow y \in F$ and $(x \rightarrow y) \rightarrow y \in F$ it follows $y \in F$, and from $y \rightarrow x \in F$ and $(y \rightarrow x) \rightarrow x \in F$ it follows $x \in F$ according to (F3). Therefore, $F$ is a prime filter in $\mathfrak{A}$.

Corollary 2. Let $\mathfrak{A}$ be a strong quasi-ordered residuated system and let $F$ and $G$ be filters in $\mathfrak{A}$ such that $F \subseteq G$. If $F$ is a prime filter that satisfies condition ( $E)$, then $G$ is a prime filter too.

Proof. Since the filter $G$ satisfies condition (E), it is a prime filter also according to Theorem 5 .

To the question 'When will a filter $F$ in a strong quasi-ordered residuated system $\mathfrak{A}$ satisfy the condition (E)?' One of the possible answers offers the following theorem.

Theorem 6. Let a strong quasi-ordered residuated system $\mathfrak{A}$ satisfy the condition

(U) $(\forall x, y \in A)((x \rightarrow y) \sqcup(y \rightarrow x)=1)$.

Then any prime filter in $\mathfrak{A}$ satisfies the condition $(E)$.

Proof. If in a strong quasi-ordered residuated system $\mathfrak{A}$ holds (U), then from $(x \rightarrow y) \sqcup(y \rightarrow x)=1 \in F$ it follows $x \rightarrow y \in F$ or $y \rightarrow x \in F$ because $F$ is a prime filter in $\mathfrak{A}$.

We will, in accordance with the practice in residuated lattices (and MTL-algebras) say that we recognize condition $(U)$ as the â€ pre-linearityâ $€^{\mathrm{TM}}$ of the system (see for example, $[19,20]$ ).

Our next theorem gives one important property of prime filters in a strong QRS. 
Theorem 7. If the relation $\preccurlyeq$ in a strong quasi-ordered residuated system $\mathfrak{A}$ is a linear relation in the following sense

$$
(\forall x, y \in A)(x \preccurlyeq y \vee y \preccurlyeq x),
$$

then every filter in $\mathfrak{A}$ is prime.

Proof. Let $x, y \in A$ be arbitrary elements. Then $x \preccurlyeq y$ or $y \preccurlyeq x$ by hypothesis. Thus $1 \preccurlyeq x \rightarrow y$ or $1 \preccurlyeq y \rightarrow x$ by (3). If $F$ is a filter in $\mathfrak{A}$, then $x \rightarrow y \in F$ or $y \rightarrow x \in F$ by (F0) and (F2). Hence $F$ is a prime filter in $\mathfrak{A}$ in accordance with (E).

Example 18. Let $\mathfrak{A}$ be as in the Example 16. The relation $\preccurlyeq$ is linear and the subset $F:=\{1\}$ is a prime filter in $\mathfrak{A}$.

One connection between the linearity of the relation $\preccurlyeq$ and the requirement that the filter $\{1\}$ be a prime filter in a strong quasi-ordered residuated system is given by the following theorem.

Theorem 8. If $\{1\}$ is a prime filter in a strong quasi-ordered residuated system $\mathfrak{A}$, then holds

$$
(\forall x, y \in A)(x \sqcup y=1 \Longrightarrow(x \preccurlyeq y \vee y \preccurlyeq x)) .
$$

Proof. Let $\{1\}$ be a prime fin $A$ and let $x, y \in A$ be such that $x \sqcup y \in\{1\}$. Then $x \in\{1\}$ or $y \in\{1\}$. Thus $y \rightarrow x \in\{1\}$ or $x \rightarrow y \in\{1\}$ by (F2). Hence $x \preccurlyeq y$ or $y \preccurlyeq x$ by (4).

Remark 5. The question remains: When will a prime filter $F$ in a strong quasi-ordered residuated system $\mathfrak{A}$ satisfy the condition (E)? When it comes to prime filters in residuated lattices, such a question is asked in the paper [19] and one answer to that question is offered in the article [20]. What should be the answer in the case of strong quasi-ordered residuated systems?

\subsection{Irreducible filters of QRS}

Definition 10. A filter $F$ of a quasi-ordered residuated system $\mathfrak{A}$ is said to be an irreducible filter in $\mathfrak{A}$ if if for any filters $S$ and $T$ of $\mathfrak{A}$ the following implication holds

(IRF) $(F=S \cap T \Longrightarrow(F=S \vee F=T))$.

Theorem 9. Any prime filter of a strong quasi-ordered residuated system is an irreducible filter.

Proof. Suppose $F$ is a prime filter of a strong quasi-ordered residuated system $\mathfrak{A}$. Let $S$ and $T$ be filters in $\mathfrak{A}$ such that $F=S \cap S$. Let us assume that the elements $s, t \in A$ be such that $s \in S$ and $t \in T$ holds. Since $s \preccurlyeq s \sqcup t$ and $t \preccurlyeq s \sqcup t$ holds, it follows $s \sqcup t \in S$ and $s \sqcup t \in T$ because $S$ and $T$ are filters in $\mathfrak{A}$. Then $s \sqcup t \in S \cap R=F$. From here, it follows $s \in F$ or $t \in F$ because $F$ is a prime filter in $\mathfrak{A}$. Since this inclusion holds for arbitrary elements $s \in S$ and $t \in T$, we conclude that $S \subseteq F$ or $T \subseteq F$ holds. As the inverse inclusion certainly holds, we conclude that $S=F$ or $T=F$ holds.

Example 19. Let $\mathfrak{A}$ be as in the Example 14. Then the subsets $\{1\},\{1, b\}$ and $\{1, c\}$ are filters in $\mathfrak{A}$ and $\{1\}=$ $\{1, b\} \cap\{1, c\}$ holds because the filter $\{1\}$ is not a prime filter.

In addition, the previous example shows that the intersection of two prime filters in a strong quasi-ordered residuated system does not have to be a prime filter.

\section{Conclusion and further work}

The concept of residuated relational systems ordered under a quasi-order relation, or quasi-ordered residuated systems (briefly, QRS), was introduced in 2018 by S. Bonzio and I. Chajda [1]. Previously, this concept was discussed in [3]. This author introduced concept of filters [4] and several types of filters in these algebraic systems such as implicative, comparative and associated filters in the articles that followed this one. It has been shown ([7]) that each comparative filter is an in implicative filter in quasi-ordered redisuated systems. In [9], the concept of strong quasi-ordered residuated systems is designed in which implicative and 
comparative filters coincide. In this paper, as a continuation of previous research, prime filters in such systems are introduced and analyzed.

In future work, for example, in addition to what has already been mentioned in Remark 5, one may investigate, among others, and the following:

- connections between prime (irreducible) and implicative filters, and

- connections between prime and irreducible filters.

Our attention regarding this class of quasi-ordered residuated systems will be devoted to the following issues:

- Is it possible to determine different types of prime filters?

- Can the requirement (IRF) in Definition 10 be weakened?

In addition to the above, research curiosity should be devoted to a strong quasi-ordered residuated system that satisfies the additional condition (U). It is an algebraic structure that we can relate to MTL-algebra for satisfying conditions (U), but it is still not an MTL-algebra since it has no common lower bound.

Acknowledgments: The author thanks the reviewer for helpful suggestions.

Conflicts of Interest: "The author declares no conflict of interest".

\section{References}

[1] Bonzio, S., \& Chajda, I. (2018). Residuated relational systems. Asian-European Journal of Mathematics, 11(2), 1850024

[2] Guido, C. (2014). Relational groupoids and residuated lattices. In: N. Galatos, A. Kurz, C. Tsinakis (eds.). TACL 2013. Sixth International Conference on Topology, Algebra and Categories in Logic, (EPiC Series, vol. 25), pp. 92-95.

[3] Bonzio, S. (2015). Algebraic structures from quantum and fuzzy logics. Ph.D Thesis. Cagliari: Universit'a degli studi di Cagliari.

[4] Romano, D. A. (2020). Filters in residuated relational system ordered under quasi-order. Bulletin of the International Mathematical Virtual Institute, 10(3), 529-534.

[5] Romano, D. A. (2021). Implicative filters in quasi-ordered residuated system. Proyecciones Journal of Mathematics, 40(2), 417-424.

[6] Romano, D. A. (2020). Associated filters in quasi-ordered residuated systems. Contributions to Mathematics, 1, $22-26$.

[7] Romano, D. A. (2021). Comparative filters in quasi-ordered residuated system. Bulletin of the International Mathematical Virtual Institute, 11(1), 177-184.

[8] Romano, D. A. (in press). Weak implicative filters in quasi-ordered residuated systems. Proyecciones Journal of Mathematics,

[9] Romano, D. A. (2021). Strong quasi-ordered residuated system. Open Journal of Mathematical Sciences, 5(1), 73-79.

[10] Raftery, J. G. (2013). Order algebraizable logics. Annals of Pure and Applied Logic, 164(3), 251-283.

[11] Raftery, J. G., \& van Alten, C. J. (2000). Residuation in commutative ordered monoids with minimal zero. Reports on Mathematical Logic, 34, 23-57.

[12] Rasiowa, H. (1974). An algebraic approach to non-classical logics. North-Holland Publishing Company, Amsterdam.

[13] Font, J. M. (1999). On special implicative filters. Mathematical Logic Quarterly (MLQ), 45(1), 117-126.

[14] Font, J. M. (2016). Abstract Algebraic Logic: An Introductory Textbook. College Publications, London.

[15] Wang, W., Yang, P., \& Xu, Y. (2019). Further complete solutions to four open problems on filter of Logical Algebras. International Journal of Computational Intelligence Systems, 12(1), 359-366.

[16] Jun, Y. B., \& Iampan, A. (2019). Implicative UP-filters. Afrika Matematika, 30(7-8), 1093-1101.

[17] Jun, Y. B., \& Iampan, A. (2019). Comparative and allied UP-filters. Lobachevskii Journal of Mathematics, 40(1), 60-66.

[18] Graätzer, G. (1998). General Lattice Theory. Birkhäuser Verlag, 1998.

[19] Van Gasse, B., Deschrijver, G., Cornelis, C., \& Kerre, E. E. (2010). Filters of residuated lattices and triangle algebras. Information Sciences, 180(16), 3006-3020.

[20] Kondo, M., \& Turunen, E. (2012). Prime filters on residuated lattices. in: IEEE 42nd International Symposium on Multiple-Valued Logic, 2012. (pp 89-91). 